EPJ Web of Conferences 64, 06004 (2014)

DOI: $10.1051 /$ epjconf/ 20146406004

(C) Owned by the authors, published by EDP Sciences, 2014

\title{
Spin period change and the magnetic fields of neutron stars in Be X-ray binaries in the SMC
}

\author{
H. Klus ${ }^{1, a}$, W.C.G. Ho ${ }^{2}$, M.J. Coe ${ }^{1}$, R.H.D. Corbet ${ }^{3}$, and L.J. Townsend ${ }^{1}$ \\ ${ }^{1}$ Physics \& Astronomy, University of Southampton, Southampton SO17 1BJ, United Kingdom \\ ${ }^{2}$ Mathematical Sciences, University of Southampton, Southampton SO17 1BJ, United Kingdom \\ ${ }^{3}$ University of Maryland Baltimore County, X-ray Astrophysics Laboratory, Mail Code 662, NASA Goddard \\ Space Flight Center, Greenbelt, MD 20771, USA
}

\begin{abstract}
We report on the long term average spin period, rate of change of spin period and X-ray luminosity during outbursts for $42 \mathrm{Be} X$-ray binary systems in the Small Magellanic Cloud. We also collect and calculate parameters of each system and use this data to determine that all systems contain a neutron star which is accreting via a disc, rather than a wind, and that if these neutron stars are near spin equilibrium, then over half of them, including all with spin periods over about 100 seconds, have magnetic fields over the quantum critical level of $4.4 \times 10^{13} \mathrm{G}$. If these neutron stars are not close to spin equilibrium, then their magnetic fields are inferred to be much lower, on the order of $10^{6}-10^{10}$ $\mathrm{G}$, comparable to the fields of neutron stars in low mass X-ray binaries. Both results are unexpected and have implications for the rate of magnetic field decay and the isolated neutron star population.
\end{abstract}

\section{Introduction}

X-ray binaries contain a compact star - a white dwarf, neutron star or black hole - and a mass donor companion. They are generally divided into two groups depending on the mass of their companion. Low mass X-ray binaries (LMXB) contain a companion comparable in mass to the Sun or less, whilst high mass X-ray binaries (HMXB) contain a companion star over ten times the mass of the Sun [1]. This is either a supergiant star - in the case of supergiant X-ray binaries (SGXB) - or an OBe star - in the case of Be X-ray binaries (BeXB). OBe stars are fast rotating $\mathrm{O}$ or B-type stars that show Balmer lines in emission, indicating the presence of a circumstellar disc. The compact star in all confirmed $\mathrm{BeXB}$ is a neutron star [2], although there are several white dwarf candidates [3, 4]. BeXB typically have eccentric orbits and at periastron the neutron star briefly passes through the edge of the OBe star's circumstellar disc where it can accrete matter, either via a Keplerian accretion disc or via a wind, causing X-ray outbursts. Once it is known how the neutron star in each system accretes then an appropriate theory of accretion can be used to determine its magnetic field.

We use archival Rossi X-ray Timing Explorer (RXTE) data taken with the Proportional Counter Array (PCA) to determine the long term average spin period, rate of change of spin period and X-ray luminosity during outbursts for $42 \mathrm{BeXB}$ in the Small Magellanic Cloud (SMC). We then determine

\footnotetext{
ae-mail: hvk1g11@ soton.ac.uk
} 
the most likely magnetic field of the neutron star in each of these systems. This is the first time that the rate of change of spin period and the long term average X-ray luminosity has been accurately measured for so many systems.

\section{Observations}

The observations used in this paper come from the study of the SMC carried out using RXTE over the period 1997-2012. The SMC was observed once or twice a week and the activity of the neutron stars determined from timing analysis. See [5] and [6] for detailed reports on this work. We remove any period detections with a significance less than $99 \%$, a collimator response less than 0.2 (with the exception of SXP15.3 which reached a high enough X-ray luminosity to compensate for the low collimator value) and datasets with less than 5 detections. This leaves 42 systems with the number of detections between 5 and 88 .

The average count rate is converted to X-ray luminosity using

$$
L=0.4 \times 10^{37} \times 3 \times C R,
$$

where we assume a distance of $60 \mathrm{kpc}$ to the SMC and an average pulsed fraction of 33\% [7]. L is the $\mathrm{X}$-ray luminosity in erg s $\mathrm{s}^{-1}$ and CR is the RXTE count rate in counts/PCU/second. We then calculate a weighted $\dot{P}$ by fitting the time evolution of the spin period using MPFITEXPR ${ }^{1}$.

All $\mathrm{H} \alpha$ measurements were obtained as part of the Southampton SMC X-ray binary pulsar (SXP) optical monitoring campaign that has been running for several years. The data were collected primarily at the SAAO $1.9 \mathrm{~m}$ telescope in South Africa and also at the ESO NTT telescope in Chile. The instrumental set-ups and the data reduction in both cases are the same as those described in [8].

The orbital periods are mostly taken from [9]. We determine the relative velocity of accreted matter from the eccentricity of the system - which is known in 6 cases and otherwise assumed to be $0.3 \pm 0.2$, the $\mathrm{H} \alpha$ equivalent width and the total mass of the system. The mass of the neutron star is assumed to be $1.4 \mathrm{M}_{\odot}$ and we determine the mass of the OBe star from spectral type and luminosity class, mostly taken from [10]. For further details see [11].

\section{Disc or Wind Accretion}

From the parameters of each system, we determine whether each neutron star is accreting via a disc or wind. An accretion disc will form if the net angular momentum per unit mass of accreted matter J, is too large for it to accrete spherically or quasi-spherically. This occurs at the circularisation radius $\mathrm{R}_{\text {circ }}$, where

$$
R_{\text {circ }}=\frac{J^{2}}{G M} \text {. }
$$

Here $\mathrm{G}$ is the gravitational constant and $\mathrm{M}$ is the mass of the neutron star. If the neutron star and its magnetosphere are fully engulfed in the OBe star's circumstellar disc then

$$
J=-\frac{1}{4}\left(n_{\rho}+1 / 2\right) V_{\text {rel }} \frac{R_{B}^{2}}{a} .
$$

Here $\mathrm{V}_{\text {rel }}$ is the relative velocity of accreted matter, $\mathrm{n}_{\rho}$ depends on the density gradient and is taken to be $2.5 \pm 0.5$ and a is the semi-major axis of the system. $R_{B}$ is the Bondi radius. In order for matter to

\footnotetext{
${ }^{1}$ www.physics.wisc.edu/ craigm/idl/down/mpfitexpr.pro
} 
be accreted it must first penetrate the neutron star's magnetosphere. The radius of the neutron star's magnetosphere is approximately equal to the Alfvén radius $\mathrm{R}_{\mathrm{A}}$ and is given by

$$
R_{A}=\left(\frac{\mu^{4}}{2 G M \dot{M}^{2}}\right)^{1 / 7} .
$$

Here $\mu$ is the magnetic moment of the neutron star $\left(\sim \mathrm{BR}_{\mathrm{NS}}{ }^{3}\right)$ where $\mathrm{R}_{\mathrm{NS}}$ is the radius of the neutron star, assumed throughout to be $10 \mathrm{~km}$. We assume a magnetic field in the range $10^{7}-10^{15} \mathrm{G}$, corresponding to $\mu \approx 10^{25}-10^{33} \mathrm{G} \mathrm{cm}^{3} . \dot{M}$ (=LR $\left.\mathrm{LR}_{\mathrm{NS}} / \mathrm{GM}\right)$ is the mass accretion rate. Thus disc accretion occurs if $\mathrm{R}_{\text {circ }}>\mathrm{R}_{\mathrm{A}}$. This inequality can be rearranged to find the maximum relative velocity of accreted matter under-which disc accretion can take place $\mathrm{V}_{\text {Crel }}$.

We determine the actual relative velocity of each system using

$$
V_{\text {rel }}=\sqrt{V_{\mathrm{w}}^{2}+V_{\text {orb }}^{2}+2 V_{\mathrm{w}} V_{\text {orb }} \cos \theta}
$$

where $\mathrm{V}_{\mathrm{w}}$ is the velocity of accreted material, which we calculate by determining the stellar wind velocity at the radius of the OBe star's circumstellar disc, $R_{c d}$. $V_{\text {orb }}$ is the orbital velocity of the neutron star and $\theta$ is the angle at which the accreted material and neutron star impact, where $\theta=180$ degrees indicates that the star and disc are in prograde motion. $\mathrm{V}_{\mathrm{w}}$ is calculated by assuming that the circumstellar disc is in a circular orbit where the radius of the disc is calculated using the $\mathrm{H} \alpha$ equivalent width [for more details see [11].] $\mathrm{V}_{\text {orb }}$ is calculated using

$$
V_{o r b}=\sqrt{\frac{G\left(M+M_{O B}\right)}{a} \frac{1+e}{1-e}},
$$

where $\mathrm{e}$ is the eccentricity of the system.

The critical relative velocity for disc accretion, $\mathrm{V}_{\mathrm{Crel}}$ and the actual relative velocity of each system, $\mathrm{V}_{\text {rel }}$ are used to determine which systems in our dataset contain neutron stars that accrete via a disc and which accrete via a quasi-spherical wind.

\section{Magnetic Fields}

We used five models to determine the magnetic fields of the neutron star in each system; the Kluzniak and Rappaport model [12], the Ghosh and Lamb model [13], the equilibrium period model for disc accretion [14, 15], the equilibrium period model for wind accretion [16, 17] and the Shakura et al. model [18]. The first three models are applicable to systems that are disc accreting and the third and fourth are applicable to systems accreting from a wind. Only the Kluzniak and Rappaport and the Ghosh and Lamb models take $\dot{P}$ into account, with the other models assuming spin equilibrium. For more details see [11] and for spin equilibrium methods see also [19].

\section{Results and discussion}

We determine the long term average $\mathrm{X}$-ray luminosity during outbursts, spin period and rate of change of spin period for $42 \mathrm{BeXB}$ systems in the SMC. A positive correlation is found between $\dot{P}$ and $\mathrm{P}$ which follows a power-law with a slope of $\sim 4 / 3$, as is shown in Figure 1 . We also determine that all prograde systems are expected to contain neutron stars which accrete via a disc [for more details see [11]]. 


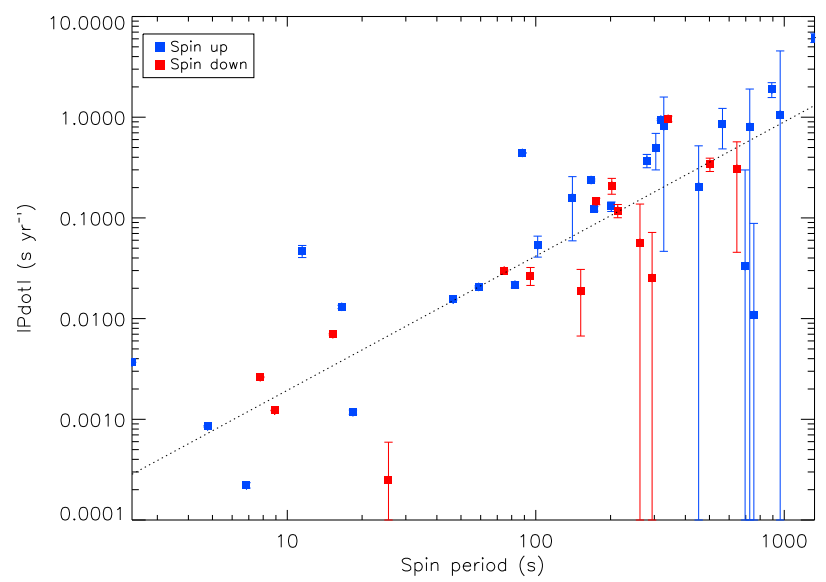

Figure 1. The long term average rate of change of spin period $\dot{P}$, against spin period $\mathrm{P}$, for neutron stars in the $42 \mathrm{BeXB}$ in our dataset: blue for $\dot{P}<0$ (spin up) and red for $\dot{P}>0$ (spin down). The dotted line indicates a correlation of $\dot{P} \propto \mathrm{P}^{4 / 3}$.

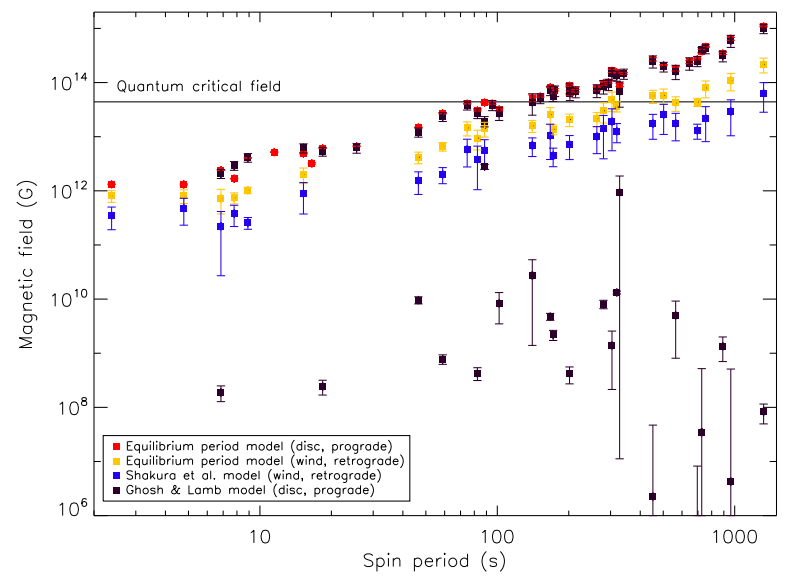

Figure 2. Magnetic field versus spin period for the neutron stars in BeXB in our dataset.

Figure 2 shows the magnetic field which we calculate using the models discussed in section 4 [excluding results from the Kluzniak and Rappaport model, for these results see [11]] where for wind accretion models we assumed retrograde motion. All models for disc accretion that assume the neutron star is close to spin equilibrium predict magnetic fields over the quantum critical level of $4.4 \times 10^{13} \mathrm{G}$ for all systems containing neutron stars with spin periods over about 100 seconds. This is over half of the systems in our dataset. The Ghosh and Lamb model can give two possible magnetic fields for stars that are spinning up, where the higher field indicates that the neutron star is closer to 
spin equilibrium. The lower fields are all well below the quantum critical value and are similar to those of neutron stars in LMXB.

The higher results may be more likely since similarly high estimates for the magnetic fields of neutron stars in binaries have been made before (see for example [20, 21]) but both of these possibilities are unexpected when compared to the magnetic fields of neutron stars in Galactic BeXB measured using CRSF. [for further discussion of the systems in our dataset in relation to other neutron star populations, see [22]].

Twelve Galactic BeXB contain neutron stars which have had their magnetic fields measured using cyclotron features. All have fields between $10^{12}-10^{13} \mathrm{G}$ and they do not show the same correlation between magnetic field and spin period that would be expected for systems containing a neutron star in spin equilibrium.

For the sources whose magnetic field has been measured by CRSF, we can use their known values of $\mathrm{P}, \mathrm{L}$ and $\dot{P}$ and the models discussed in Section 4 to cross-check this magnetic field. This is currently possible for five sources: GRO J1008-57 [23], A0535+26 [24, 25], RX J0440.9+4431 [26], 1A1118$616[27,28]$ and X Per [29]. Assuming that the neutron stars in these systems are disc accreting and close to spin equilibrium, then all models predict higher fields than those determined by the cyclotron features.

If the magnetic fields predicted by the Ghosh and Lamb model are taken to be the magnetic field at the surface of the neutron star and the CRSF gives the magnetic field of the accretion column, then the column would have to be $4-30 \mathrm{~km}$ above the surface (assuming $B \propto r^{-3}$ ). A similar difference in field determination has previously been noted for SGXB GX 301-2 [30].

The heights of the accretion column in these systems can be calculated using the equations given by [31] and these are between $\sim 0.1$ and $2 \mathrm{~km}$ from the surface except in the case of $\mathrm{X}$ Per where the height is $\sim 9 \mathrm{~km}$ from the surface. In order to obtain the required height to reconcile the two magnetic field determinations for each source, the X-ray luminosity of the neutron star must be less then $\sim 10^{34} \mathrm{erg} \mathrm{s}^{-1}$. However [32] suggest that this issue may be more complex, with the CRSF from a single source changing due to the fact that the CRSF can originate from radiation that is produced by the accretion column and reflected from the neutron star's surface rather than from an accretion column that is changing heights. Although it is currently unclear why the values predicted using the Ghosh and Lamb model are so different to those determined by cyclotron features, future work in this area would help resolve the matter.

If over half the neutron stars in systems in our dataset do have fields over the quantum critical value then this could mean that magnetic field decay occurs more slowly than previously thought [33], it may also mean that half the isolated neutron star population also have fields this high.

\section{Acknowledgements}

HK acknowledges a studentship from the Science and Technology Facilities Council (STFC) in the United Kingdom. WCGH acknowledges support from STFC. LJT acknowledges support from the University of Southampton Mayflower scholarship.

\section{References}

[1] Grimm, H.J., Gilfanov, M. \& Sunyaev, R., MNRAS, 339, 793, (2003)

[2] Reig, P., Ap\&SS, 332, 1, (2011)

[3] Haberl, F., A\&A, 296, 685, (1995) 
[4] de Oliveira, R.L., Motch, C., Haberl, F., Negueruela, I., \& Janot-Pacheco, E., A\&A, 454, 265, (2006)

[5] Laycock, S., Corbet, R.H.D., Coe, M.J., Marshall, F.E., Markwardt, C. \& Lochner, J., ApJS, 161, 96, (2005)

[6] Galache, J.L., Corbet, R.H.D., Coe, M.J., Laycock, S., Schurch, M.P.E., Markwardt, C., Marshall, F.E. \& Lochner, J., ApJS, 177, 189, (2008)

[7] Coe, M.J., McBride, V.A. \& Corbet, R.H.D., MNRAS, 401, 252, (2010)

[8] Coe, M.J., Haberl, F., Sturm, R., Bartlett, E.S., Hatzidimitriou, D., Townsend, L.J., Udalski, A., Mereghetti, S. \& Filipovic, M., MNRAS, 424, 282, (2012)

[9] Bird, A. J., Coe, M. J., McBride, V. A. \& Udalski, A., MNRAS, 423, 3663, (2012)

[10] McBride, V.A., M.J. Coe, I. Negueruela, I., Schurch, M. P. E. \& McGowan, K. E., MNRAS, 388, 1198, (2008)

[11] Klus, H., Ho, W.C.G., Coe, M.J., Corbet, R.H.D. and Townsend, L.J., MNRAS, submitted, (2013)

[12] Kluzniak, W., \& Rappaport, S., AJ, 671, 1990, (2007)

[13] Ghosh, P., \& Lamb, F.K., AJ, 234, 296, (1979)

[14] Davidson, K., \& Ostriker, J. P., AJ, 179, 585, (1973)

[15] Alpar, M.A., Cheng, A.F., Ruderman, M.A. \& Shaham, J., Nature, 300, 728, (1982)

[16] Illarionov, A.F. \& Sunyaev, R.A., A\&A, 39, 185, (1975)

[17] Illarionov, A.F. \& Kompaneets, D.A., MNRAS, 247, 219, (1990)

[18] Shakura, N., Postnov, K., Kochetkova, A., \& Hjalmarsdotter, L., MNRAS, 420, 216, (2012)

[19] Chashkina, A. \& Popov, S.B, New Astronomy, 17, 594, (2012)

[20] Bozzo, E., Falanga, M., \& Stella, L., AJ, 683, 1031, (2008)

[21] Reig, P., Torrejón, J. M., \& Blay, P., MNRAS, 425, 595, (2012)

[22] Ho, W.C.G., Klus, H., Coe, M.J. \& Andersson, N., MNRAS, Submitted, (2013)

[23] Shrader, C.R., Sutaria, F.K., Singh, K.P. \& Macomb, D.J., ApJ, 512, 920, (1991)

[24] Kendziorra, E., Kretschmar, P., Pan, H.C., Kunz, M., Maisack, M., Staubert, R., Pietsch, W., Truemper, J., Efremov, V. \& Sunyaev, R., A\&A, 291, L31, (1994).

[25] Maisack, M., Grove, J.E., Kendziorra, E., Kretschmar, P., Staubert, R., Strickman, M.S., A\&A, 325, 212, (1997)

[26] Tsygankov, S.S., Krivonos, R.A. \& Lutovinov, A.A., MNRAS, 421, 2407, (2012)

[27] Doroshenko, V., Suchy, S., Santangelo, A., Staubert, R., Kreykenbohm, I., Rothschild, R., Pottschmidt, K. \& Wilms, J., A\&A, 515, L1, (2010a)

[28] Nespoli, E., \& Reig., P., A\&A, 526, 7, (2011)

[29] Coburn, W., Heindl, W.A., Gruber, D.E., Rothschild, R.E., Staubert, R., Wilms, J., Kreykenbohm, I., ApJ, 552, 738, (2001)

[30] Doroshenko, V., Santangelo, A., Suleimanov, V., Staubert, R., Kreykenbohm, I., Ferrigno, C., \& Klochkov. D., AIP Conference Proceedings, 1248, 155, (2010b)

[31] Becker, P.A., Klochkov, D., Schonherr, G., Nishimura, O., Ferrigno, C., Caballero, I., Kretschmar, P., Wolff, M. T., Wilms, J. \& Staubert, R., A\&A, 544, A123, (2012)

[32] Poutanen, J., Mushtukov, A.A., Suleimanov, V.F., Tsygankov, S.S., Nagirner, D.I., Doroshenko, V., \& Lutovinov, A.A., ApJ, 777, 115 (2013)

[33] Pons, J.A., Miralles, J.A. \& Geppert, U., A\&A, 496, 207, (2009) 